\title{
POISSON INTEGRALS FOR HERMITE AND LAGUERRE EXPANSIONS
}

\author{
BY \\ BENJAMIN MUCKENHOUPT $\left(^{(}\right)$
}

1. Introduction. Professor E. M. Stein suggested that many of the results in [3] would have analogues in the theory of Hermite and Laguerre expansions. As is shown in this paper this is indeed the case for theorems concerning the convergence of "Poisson integrals". The principal result is that the Poisson integrals in these cases are majorized by suitable Hardy maximal functions. This implies standard $L^{p}$ results and the convergence almost everywhere to the original function.

As it turned out, a strict imitation of the methods in [3] to prove convergence almost everywhere would have produced prohibitively long and involved proofs. To avoid this, a theorem proved by Natanson, [4, p. 16], is generalized in $\S 2$; this would be equally useful for simplifying proofs in [3] and elsewhere. It states that the integral of a product of two functions is less than an appropriate Hardy maximal function of the first function at a point if the second function is monotone decreasing above this point, monotone increasing below this point and has integral one. This permits an easy estimation of integrals that in the classical Fourier series case are convolutions but in the generalizations have variable kernels.

The obvious Poisson integral for a function $f(y)$ with Hermite expansion $\sum a_{n} H_{n}(y)$ is the function $g(r, y)$ with Hermite expansion $\sum r^{n} a_{n} H_{n}(y), 0 \leqq r<1$. Merely defining $g$ to be the sum of this series is unsatisfactory since, as observed in $\S 3$, the series may diverge for some values of $r$ less than 1 . An integral definition is more satisfactory since it is usable even for functions in $L^{1}\left(\exp \left(-y^{2}\right)\right)$ that have no Hermite expansion. In $\S 3$ it is shown that this Poisson integral is maximized by a suitable Hardy maximal function and that it converges almost everywhere as $r$ approaches $1^{-}$. The usual estimates concerning the norm of $\sup _{r \rightarrow 1} g(r, y)$ are also proved for $f \in L^{p}\left(\exp \left(-y^{2}\right)\right), p>1$. All these results are a fairly straightforward application of the general theorem in $\S 2$. An alternate Poisson integral, $f(x, y)$, is also mentioned. If $f(y)$ has the Hermite expansion given above, $f(x, y)$ is the function which for fixed $x>0$ has the expansion $\sum a_{n} \exp \left[-(2 n)^{1 / 2} x\right] H_{n}(y)$. The theorems proved for $g$ are immediately applicable to this since there is a simple relation between it and $g$. Like the ordinary Poisson integral, $f(x, y)$ satisfies a second order elliptic differential equation. In fact, $f_{11}(x, y)+f_{22}(x, y)-2 y f_{2}(x, y)=0$. This makes $f(x, y)$ a more reasonable Poisson integral and makes it possible to

Received by the editors April 3, 1967.

( ${ }^{1}$ ) Supported in part by NSF Grant GP 4219. 
define conjugate functions for Hermite expansions. These conjugate functions will be treated in another paper.

For the Laguerre case, if $f(y)$ has the expansion $\sum a_{n} L_{n}^{\alpha}(y)$, the first Poisson integral considered is the function $g(r, y)$ with Laguerre expansion $\sum \cdot r^{n} a_{n} L_{n}^{\alpha}(y)$. Again an integral definition is used and the same sorts of theorems are proved in $\S 4$ as in $\$ 3$ for the Hermite case. The formulas are more complicated because they contain Bessel functions, but the principles of $\$ 2$ still apply. Again, an alternate Poisson integral, $f(x, y)$, is defined. If $f(y)$ has the Laguerre expansion given above, $f(x, y)$ is the function which for fixed $x>0$ has the expansion $\sum a_{n} \exp (-\sqrt{ } n x) L_{n}^{\alpha}(y)$. Then, $f_{11}(x, y)+y f_{22}(x, y)+(\alpha+1-y) f_{2}(x, y)=0$. As before this makes $f(x, y)$ a more reasonable Poisson integral and makes it possible to define conjugate functions for Laguerre expansions.

2. A majorization theorem. For this section there will be assumed given a fixed interval $I$ and an absolutely continuous finite measure, $\mu$, on $I$. As usual the symbol $\|f\|_{p}$ will be used for

$$
\left(\int_{I}|f(y)|^{p} d \mu(y)\right)^{1 / p}, \quad 1 \leqq p<\infty
$$

and $\|f\|_{\infty}$ will be ess sup $|f|$. Given a function $f(y)$ in $L^{1}(d \mu)$, it is appropriate to consider the analogue of the Hardy maximal function, $f^{*}(y)$, defined by

$$
f^{*}(y)=\sup _{y \neq z ; y, z \in I}\left(\int_{y}^{z}|f(y)| d \mu(y) / \int_{y}^{z} d \mu(y)\right) .
$$

Lemma 2 on p. 28 of [3] then has the following generalization.

LEMMA 1. If $\mu$ is an absolutely continuous finite measure on an interval I and $f(y)$ is in $L^{1}(d \mu)$, then $f^{*}(y)$ is finite for almost every $y$. For any $a>0$, let $E_{a}=\left\{y \mid f^{*}(y)>a\right\}$. Then $\mu\left(E_{a}\right) \leqq(2 / a) \int_{I}|f| d \mu$. If in addition $f \in L^{p}(d \mu), 1<p \leqq \infty$, then $f^{*} \in L^{p}(d \mu)$ and $\left\|f^{*}\right\|_{p} \leqq A_{p}\|f\|_{p}$.

The proof of this is an exact repetition of the one given for Lemma 2 in [3].

The main theorem of this section, a generalization of a result in $[4, p .16]$, can now be stated.

THEOREM 1. If $\mu$ is an absolutely continuous measure on an interval $I, f$ and $g$ are in $L^{1}(d \mu)$, and $g(z)$ is nonnegative, monotone increasing for $z \leqq y$ and monotone decreasing for $z \geqq y$, then $\left|\int_{I} f g d \mu\right| \leqq\|g\|_{1} f^{*}(y)$.

Let $\chi_{E}$ denote the characteristic function of the set $E$. If $g$ is a simple function, then except possibly for a finite number of points it can be written in the form $\sum a_{i} \chi_{\left[y, z_{i}\right]}+\sum b_{j} \chi_{\left[x_{j}, y\right]}$ where the $a_{i}$ and $b_{j}$ are positive. Substituting this for $g$ shows that

$$
\left|\int_{I} f g d \mu\right| \leqq \sum_{i} a_{i} \mu\left(\left[y, z_{i}\right]\right) f^{*}(y)+\sum_{j} b_{j} \mu\left(\left[x_{j}, y\right]\right) f^{*}(y)
$$

since for an interval $J$ with one end at $y,\left|\int_{J} f d \mu\right| \leqq \mu(J) f^{*}(y)$. The right side of (2.2), however, is just $\|g\|_{1} f^{*}(y)$. For the general case choose a monotone increasing 
sequence of nonnegative simple functions, $g_{n}$, which are monotone increasing for $z \leqq y$, monotone decreasing for $z \geqq y$ and converge to $g$ pointwise. Then using the result just proved and the monotone convergence theorem twice gives

$$
\left|\int_{I} f g d \mu\right| \leqq \int_{I}|f g| d \mu=\lim _{n \rightarrow \infty} \int_{I}|f| g_{n} d \mu \leqq \lim _{n \rightarrow \infty} f^{*}(y)\left\|g_{n}\right\|_{1}=f^{*}(y)\|g\|_{1} .
$$

This leads to the following corollaries that are the form in which this theorem will be used.

COROLlary 1. Let $\mu$ be an absolutely continuous finite measure on an interval I. Let $L(y, z)$ be a nonnegative function, monotone increasing in $z$ for $z \leqq y$, monotone decreasing in $z$ for $z \geqq y$, and $\int_{I} L(y, z) d \mu(z) \leqq B$ where $B$ is independent of $y$. Let $f(z)$ be in $L^{1}(d \mu)$ and $g(y)=\int_{I} L(y, z) f(z) d \mu(z)$. Then $|g(y)| \leqq B f^{*}(y)$ and

$$
\mu(\{y|| g(y) \mid>a\}) \leqq \frac{2 B}{a} \int_{I}|f| d \mu .
$$

Furthermore, if $f \in L^{p}(d \mu)$, then $\|g\|_{p} \leqq B A_{p}\|f\|_{p}$ where $A_{p}$ depends only on $p$.

The first conclusion is just Theorem 1 , and the rest are immediate consequences of Lemma 1.

Unfortunately, the kernels to be considered usually do not have the desired monotonicity properties. The following modification then is usually the most useful.

Corollary 2. Let $\mu, L, B$ and $f$ satisfy the hypotheses of Corollary 1 and $|K(y, z)| \leqq L(y, z)$. 'Then the conclusions of Corollary 1 are valid for the function $h(y)=\int_{I} K(y, z) f(z) d \mu(z)$.

This follows from the facts that $|f|^{*}$ is the same as $f^{*}$ and

$$
h(y) \leqq \int_{I}|K(y, z)||f(z)| d \mu(z) \leqq \int_{I} L(y, z)|f(z)| d \mu(z) .
$$

3. Poisson integrals for Hermite expansions. If $f(y)$ is in $L^{1}\left(\exp \left(-y^{2}\right)\right)$, define its Poisson integral, $g(r, y)$, for $0 \leqq r<1$ by

$$
g(r, y)=\int_{-\infty}^{\infty} P(r, y, z) f(z) \exp \left(-z^{2}\right) d z
$$

where

$$
P(r, y, z)=\sum_{n=0}^{\infty} \frac{r^{n} H_{n}(y) H_{n}(z)}{\pi^{1 / 2} 2^{n} n !}
$$

The definition of the $n$th Hermite polynomial, $H_{n}$, used here will be the one used in [ $]$ ]. As shown in [2, p. 192], $P$ may also be written as

$$
P\left(r^{\prime}, y, z\right)=\frac{1}{\pi^{1 / 2}\left(1-r^{2}\right)^{1 / 2}} \exp \left(\frac{-r^{2} y^{2}+2 r y z-r^{2} z^{2}}{1-r^{2}}\right) .
$$


It should be noted that for a fixed $y$ and $r$ the maximum value of $P(r, y, z)$ is $\exp \left(y^{2}\right) /\left(\pi\left(1-r^{2}\right)\right)^{1 / 2}$. Consequently, $g(r, y)$ exists for every $f(y)$ in $L^{1}\left(\exp \left(-y^{2}\right)\right)$ whether or not it has a Hermite expansion. The relationship between various possible definitions of $g$ is given in the following lemma.

Lemma 2. If $f(y)$ has the Hermite expansion $\sum a_{n} H_{n}(y)$, then for each $r, 0 \leqq r<1$, $g(r, y)$ has the Hermite expansion $\sum r^{n} a_{n} H_{n}(y)$. If $f(y)$ is in $L^{2}\left(\exp \left(-y^{2}\right)\right)$, then $\sum r^{n} a_{n} H_{n}(y)$ converges absolutely to $g(r, y)$ almost everywhere. For every $p, 1 \leqq p<2$, there exists a function $f(y)$ in $L^{p}\left(\exp \left(-y^{2}\right)\right)$ and an $r<1$ such that $\sum r^{n} a_{n} H_{n}(y) d i-$ verges for every $y$.

Here and hereafter let $C$ denote a constant not necessarily the same at each occurrence. For a fixed $z$, by [6, p. $198(8.22 .8)]$,

$$
\left|H_{n}(z)\right| \leqq C n ! / \Gamma\left(\frac{1}{2} n+1\right) .
$$

By Hölder's inequality

$$
\int_{-\infty}^{\infty}\left|H_{n}(y) H_{k}(y)\right| \exp \left(-y^{2}\right) d y \leqq\left\|H_{n}(y)\right\|_{2}\left\|H_{k}(y)\right\|_{2}
$$

where \|\| denotes the norm with weight function $\exp \left(-y^{2}\right)$. Combining these facts, the value of $\left\|H_{n}(y)\right\|_{2}^{2}=\pi^{1 / 2} 2^{n} n$ ! from [6, p. 104 (5.5.1)], and Stirling's formula then gives for a fixed $z$ the fact that

$$
\int_{-\infty}^{\infty} \frac{r^{n}}{\pi^{1 / 2} 2^{n} n !}\left|H_{n}(y) H_{k}(y) H_{n}(z)\right| \exp \left(-y^{2}\right) d y \leqq C r^{n}\left\|H_{k}(y)\right\|_{2} .
$$

This and the dominated convergence theorem justify integrating the right side of (3.2) multiplied by $H_{k}(y)$ term by term. This produces

$$
\int_{-\infty}^{\infty} P(r, y, z) H_{k}(y) \exp \left(-y^{2}\right) d y=r^{k} H_{k}(z)
$$

provided that $|r|<1$. That

$$
\int_{-\infty}^{\infty} g(r, y) H_{n}(y) \exp \left(-y^{2}\right) d y=\pi^{1 / 2} 2^{n} n ! a_{n} r^{n},
$$

is obtained by substituting (3.1) into the left side, interchanging the order of integration and using (3.6). This completes the first part of the lemma.

To prove the second part, observe that by use of Hölder's inequality, $\left|a_{n}\right|$ $\leqq\left\|H_{n}\right\|_{2}\|f\|_{2} / \pi^{1 / 2} 2^{n} n$ !. Using (3.4) and Stirling's formula then shows that $a_{n} H_{n}(y)$ is a bounded sequence for each $y$. Therefore, $\sum r^{n} a_{n} H_{n}(y)$ must converge for every $y$ if $0 \leqq r<1$. Since by standard $L^{2}$ theory it must converge to $g(r, y)$ in norm, its pointwise limit is also $g(r, y)$ almost everywhere.

For the third part use is made of an example of Pollard in [5, p. 366]. He showed that given any $p<2$, there is a function in $L^{p}\left(\exp \left(-y^{2}\right)\right)$ such that

$$
\lim \sup \left(\left|a_{n} H_{n}(y)\right|\right)^{1 / n}
$$


is a fixed number greater than 1 for every $y$. For $r$ sufficiently close to 1 , then, the series expansion for $g$ diverges for every $y$.

To apply Corollary 2 to $g(r, y)$ the following lemma is needed.

LeMma 3. Let $L(r, y, z)$ equal $P(r, y, y / r)$ for $y \leqq z \leqq y / r$ and equal $P(r, y, z)$ for $z$ elsewhere. Then for each $r, 0 \leqq r<1, L(r, y, z)$ is monotone increasing in $z$ for $z \leqq y$, monotone decreasing for $z \geqq y$ and $\int_{-\infty}^{\infty} L(r, y, z) \exp \left(-z^{2}\right) d z \leqq 3$.

Simple calculus shows that $P(r, y, z)$ is monotone increasing in $z$ for $z \leqq y / r$ and monotone decreasing in $z$ for $z \geqq y / r$. This immediately gives the desired monotonicity properties for $L$. Setting $k=0$ in (3.6) and using the fact that $P(r, y, z)$ is symmetric in $y$ and $z$ gives

$$
\int_{-\infty}^{\infty} P(r, y, z) \exp \left(-z^{2}\right) d z=1 .
$$

Because of this and the fact that $P(r, y, y / r)=\exp \left(y^{2}\right) /\left(\pi\left(1-r^{2}\right)\right)^{1 / 2}$, the integral inequality may be proved by showing that

$$
\exp \left(y^{2}\right) /\left(\pi\left(1-r^{2}\right)\right)^{1 / 2}\left|\int_{y}^{y / r} \exp \left(-z^{2}\right) d z\right| \leqq 2 .
$$

It is sufficient to consider only $y \geqq 0$; the proof of (3.8) will be split into 3 cases.

Case 1. If $r \geqq\left(1+y^{2}\right) /\left(2+y^{2}\right)$, the integral in (3.8) is less than $(y / r-y) \exp \left(-y^{2}\right)$ and the product is less than

$$
\frac{y}{r} \frac{(1-r)^{1 / 2}}{(1+r)^{1 / 2}} \leqq 2 y(1-r)^{1 / 2}
$$

since $r \geqq 1 / 2$. Then $2 y(1-r)^{1 / 2} \leqq 2 y /\left(2+y^{2}\right)^{1 / 2} \leqq 2$.

Case 2. If $r<\left(1+y^{2}\right) /\left(2+y^{2}\right)$ and $y<1$, then integral is less than $\frac{1}{2} \sqrt{ } \pi$ and $r<2 / 3$. The product then is less than $e / 2(5 / 9)^{1 / 2} \leqq 2$.

Case 3. If $r<\left(1+y^{2}\right) /\left(2+y^{2}\right)$ and $y \geqq 1$, the integral is less than

$$
\sum_{k=0}^{\infty} \int_{y+k / y}^{y+(k+1) / y} \exp \left(-z^{2}\right) d z<\sum_{k=0} \frac{\exp \left(-y^{2}-2 k\right)}{y} \leqq \frac{\exp \left(-y^{2}\right)}{y\left(1-e^{-2}\right)} .
$$

The product then is less than

$$
\frac{1}{\pi^{1 / 2} y\left(1-e^{-2}\right)(1-r)^{1 / 2}} \leqq \frac{2\left(2+y^{2}\right)^{1 / 2}}{3 y} \leqq 2 .
$$

The main theorem about Poisson integrals for Hermite expansions may now be stated.

THEOREM 2. Let $f \in L^{p}\left(\exp \left(-y^{2}\right)\right), 1 \leqq p \leqq \infty$, let $g(r, y)$ denote its Poisson integral (3.1), and $f^{*}(y)$ the function (2.1) with $d \mu(y)=e^{-y^{2}} d y$ and $I=(-\infty, \infty)$. Then if $0 \leqq r<1$, 
(a) $|g(r, y)| \leqq 3 f^{*}(y)$,

(b) $\|g(r, y)\|_{p} \leqq\|f(y)\|_{p}, 1 \leqq p \leqq \infty$,

(c) $\|g(r, y)-f(y)\|_{p} \rightarrow 0$ as $r \rightarrow 1^{-}$for $1 \leqq p<\infty$,

(d) $\lim _{r \rightarrow 1}-g(r, y)=f(y)$ almost everywhere, $1 \leqq p \leqq \infty$,

(e) $\left\|\sup _{r<1} g(r, y)\right\|_{p} \leqq A_{p}\|f\|_{p}, 1<p \leqq \infty$.

Part (a) is an immediate consequence of Lemma 3 and Corollary 2. Part (e) follows from part (a) and Lemma 1. Part (b) is proved using (3.7) in the same way that part (a) of Theorem 2, p. 31 of [3] was proved.

To prove parts (c) and (d), consider the linear space, $S$, of continuous functions with compact support on $(-\infty, \infty)$. Then given an $f \in S$ and an $\varepsilon>0, P(r, y, z)$ converges uniformly to 0 as $r \rightarrow 1^{-}$for $|y-z|>\varepsilon$ and $z$ in the support of $f$. This is clear if $P$ is written in the form

$$
\frac{1}{\left(\pi\left(1-r^{2}\right)\right)^{1 / 2}} \exp \left(\frac{-[r(y-z)-z(1-r)]^{2}}{1-r^{2}}+z^{2}\right)
$$

since for $0 \leqq(1-r)<\varepsilon r / 2 \max z, P$ is less than

$$
\frac{1}{\left(\pi\left(1-r^{2}\right)\right)^{1 / 2}} \exp \left(\frac{-r^{2} \varepsilon^{2}}{4\left(1-r^{2}\right)}+\max z^{2}\right) \text {. }
$$

Because of (3.7), $g(r, y)$ converges to $f(y)$ if $f \in S$ and (c) and (d) follow for this case.

For the general case, (c) and (d) are proved in the same way as parts (b) and (c) of Theorem 2, p. 31 of [3] are proved from their special case. Here, use is made of (3.7) and the results proved above for $S$.

It is now a simple matter to treat the alternate Poisson integral $f(x, y)$ mentioned in the introduction. The formula

$$
e^{-\alpha}=\int_{0}^{\infty} \frac{1}{u^{2} \sqrt{ } \pi} \exp \left(\frac{-1}{4 u^{2}}\right) \exp \left(-\alpha^{2} u^{2}\right) d u
$$

obtained from [1, p. 38] with a correction will be used. Making the substitutions $\alpha=(2 n)^{1 / 2} x$ and $u=\left(-\frac{1}{2} \log r\right)^{1 / 2} / x$ produces

$$
\int_{0}^{1} T(x, r) r^{n} d r=\exp \left[-(2 n)^{1 / 2} x\right]
$$

where

$$
T(x, r)=\frac{x \exp \left(x^{2} / 2 \log r\right)}{(2 \pi)^{1 / 2} r(-\log r)^{3 / 2}} .
$$

Since $f(x, y)$ is supposed to have the expansion $\sum a_{n} \exp \left[-(2 n)^{1 / 2} x\right] H_{n}(y)$, it is clear that $f(x, y)$ should be defined by

$$
f(x, y)=\int_{0}^{1} T(x, r) g(r, y) d r=\int_{-\infty}^{\infty}\left(\int_{0}^{1} T(x, r) P(r, y, z) d r\right) f(z) \exp \left(-z^{2}\right) d z
$$


for $x>0$. For every $y$ for which $f^{*}(y)$ is finite, $g(r, y)$ is a bounded function of $r$ by part (a) of Theorem 2. $T$ is nonnegative and

$$
\int_{0}^{1} T(x, r) d r=1
$$

as shown by (3.9) with $n=0$. Therefore, for every $x, f(x, y)$ will be defined for almost every $y$ provided $f$ is in $L^{1}\left(\exp \left(-y^{2}\right)\right)$. The facts about $f(x, y)$ may be summarized as follows.

Corollary 3. If $f(y)$ has Hermite expansion $\sum a_{n} H_{n}(y)$, then for each $x>0$, $f(x, y)$ has the Hermite expansion $\sum \exp \left[-(2 n)^{1 / 2} x\right] a_{n} H_{n}(y)$. If $f(y)$ is in $L^{2}\left(\exp \left(-y^{2}\right)\right)$, then $\sum \exp \left[-(2 n)^{1 / 2} x\right] a_{n} H_{n}(y)$ converges absolutely to $f(x, y)$. The conclusions of Theorem 2 are valid if $g(r, y)$ is replaced by $f(x, y), r \rightarrow 1^{-}$by $x \rightarrow 0^{+}$ and $0 \leqq r<1$ by $x>0$. Furthermore $f_{11}(x, y)+f_{22}(x, y)-2 y f_{2}(x, y)=0$.

The first statement is proved in the same way as the first part of Lemma 2 by interchanging orders of integration. The second part is proved as it was in Lemma 2 from the boundedness of $a_{n} H_{n}(y)$ for a fixed $y$. This boundedness and the differential equation for Hermite polynomials proves the given differential equation for $f(x, y)$ if $f(y)$ is in $L^{2}$. This implies the differential equation for the kernel in (3.11), and therefore for any $f(x, y)$. Differentiating under the integral sign is justified by showing that the derivatives of the kernel are bounded in $z$ for $x$ and $y$ in a suitable neighborhood of $\left(x_{0}, y_{0}\right)$. This is easily done by estimating the maximum in $z$ of the derivatives of $T(x, r) P(r, y, z)$ and integrating with respect to $r$.

For the analogy to Theorem 2, parts (a), (b) and (e) are proved from the corresponding parts of Theorem 2 by use of Minkowski's integral inequality and (3.12). Parts (c) and (d) follow from the corresponding parts of Theorem 2 by use of (3.12) and the fact that for fixed $r, T(x, r) \rightarrow 0$ as $x \rightarrow 0^{+}$.

4. Poisson integrals for Laguerre expansions. If $f(y)$ is in $L^{1}\left(y^{\alpha} \exp (-y)\right)$, $\alpha>-1$, define its Poisson integral, $g(r, y)$, for $0 \leqq r<1$ by

$$
g(r, y)=\int_{0}^{\infty} K(r, y, z) f(z) z^{\alpha} \exp (-z) d z
$$

where

$$
K(r, y, z)=\sum_{n=0}^{\infty} r^{n} L_{n}^{\alpha}(y) L_{n}^{\alpha}(z) / \Gamma(\alpha+1)\left(\begin{array}{c}
n+\alpha \\
n
\end{array}\right) .
$$

Again, the definition of the $n$th Laguerre polynomial, $L_{n}^{\alpha}$, used here will be the one used in [6]. As stated in [6, p. 101], $K$ may also be written as

$$
K(r, y, z)=\frac{(-r y z)^{-\alpha / 2}}{1-r} \exp \left(\frac{-r(y+z)}{1-r}\right) J_{\alpha}\left(\frac{2(-r y z)^{1 / 2}}{1-r}\right)
$$

where $J_{\alpha}$ is the standard Bessel function. 
Using the expression for a Bessel function with an imaginary argument, (2), p. 77 of [7], the asymptotic expression (2), p. 203 of [7] and the monotonicity of these functions shows that there are positive constants $c$ and $C$ depending only on $\alpha$ such that

$$
\begin{array}{ll}
c w^{\alpha} \leqq i^{-\alpha} J_{\alpha}(i w) \leqq C w^{\alpha} & 0 \leqq w \leqq 1 ; \\
c w^{-1 / 2} e^{w} \leqq i^{-\alpha} J_{\alpha}(i w) \leqq C w^{-1 / 2} e^{w} & 1 \leqq w .
\end{array}
$$

Using (4.4) in (4.3), it is clear that there exist positive constants $c$ and $C$ depending only on $\alpha$ such that

$$
c H(r, y, z) \leqq K(r, y, z) \leqq C H(r, y, z)
$$

where

$$
\begin{aligned}
H(r, y, z) & =(1-r)^{-\alpha-1} \exp \left(\frac{-r(y+z)}{1-r}\right), & & 0 \leqq z<\frac{(1-r)^{2}}{4 r y}, \\
& =\frac{(4 r y z)^{-\alpha / 2-1 / 4}}{e(1-r)^{1 / 2}} \exp \left(\frac{-r y+2(r y z)^{1 / 2}-r z}{1-r}\right), & & \frac{(1-r)^{2}}{4 r y} \leqq z .
\end{aligned}
$$

For a fixed $y, r$ and $\alpha, H$ is a bounded function of $z$. Therefore, as in the Hermite case, $g(r, y)$ exists for every $f(y)$ in $L^{1}\left(y^{\alpha} e^{-y}\right)$ whether or not it has a Laguerre expansion. The relationship between various possible definitions of $g$ is given in the following Laguerre version of Lemma 2.

LEMMA 4. If $f(y)$ has the Laguerre expansion $\sum a_{n} L_{n}^{\alpha}(y)$, then for each $r, 0 \leqq r<1$, $g(r, y)$ has the Laguerre expansion $\sum r^{n} a_{n} L_{n}^{\alpha}(y)$. If $f(y)$ is in $L^{2}\left(y^{\alpha} e^{-y}\right)$, then

$$
\sum r^{n} a_{n} L_{n}^{\alpha}(y)
$$

converges absolutely to $g(r, y)$ almost everywhere. For every $p, 1 \leqq p<2$, there exists a function $f(y)$ in $L^{p}\left(y^{\alpha} e^{-y}\right)$ and an $r<1$ such that $\sum r^{n} a_{n} L_{n}^{\alpha}(y)$ diverges for every $y$.

For a fixed $\alpha$ and $z$, by [6, p. $197(8.22 .6)],\left|L_{n}^{\alpha}(z)\right| \leqq C n^{\alpha / 2-1 / 4}$. Then using (5.7.16), p. 109 of [6], produces the analogy to (3.5). The reasoning of Lemma 2 then produces

$$
\int_{0}^{\infty} K(r, y, z) L_{n}^{\alpha}(y) e^{-y} y^{\alpha} d y=r^{n} L_{n}^{\alpha}(z)
$$

The proof of the first two parts of Lemma 4 then is the same as the proof for the first two parts of Lemma 2. The last part is proved in the same way as for Lemma 2, using the example sketched by Pollard on p. 367 of [5].

To prove the main theorem of this section the following lemma is needed.

LEMMA 5. There is a function, $L(r, y, z)$, such that for each $r, 0 \leqq r<1, L(r, y, z)$ is monotone increasing in $z$ for $z \leqq y$, monotone decreasing for $z \geqq y$ and $H(r, y, z)$ $\leqq L(r, y, z)$. Furthermore, there is a constant $C$ independent of $r$ and $y$ such that $\int_{0}^{\infty} L(r, y, z) e^{-z} z^{\alpha} d z \leqq C$. 
Define $L(r, y, z)$ for each $z$ to be the largest of $H(r, y, z)$ and any maximums of $H(r, y, t)$ that occur for $t$ on the opposite side of $z$ from $y$. Then $L$ clearly has the desired monotonicity properties and it remains to prove the boundedness of the integral.

Putting $n=0$ in (4.7) and using the fact that $K(r, y, z)$ is symmetric in $y$ and $z$ gives

$$
\int_{0}^{\infty} K(r, y, z) e^{-z} z^{\alpha} d z=1
$$

Using (4.5) then there is a constant $C$ such that

$$
\int_{0}^{\infty} H(r, y, z) e^{-z} z^{\alpha} d z \leqq C .
$$

Following the method of proof of Lemma 3, it will be necessary to consider for a fixed $r$ and $y$ the values $m_{1}, m_{2}, \ldots$ of $z$ for which $H(r, y, z)$ takes on a maximum. With this notation it is clear that

$$
\int_{0}^{\infty} L(r, y, z) e^{-z} z^{\alpha} d z \leqq \int_{0}^{\infty} H(r, y, z) e^{-z} z^{\alpha} d z+\sum_{i} H\left(r, y, m_{i}\right)\left|\int_{y}^{m_{i}} e^{-z} z^{\alpha} d z\right| .
$$

Since (4.9) gives a bound for the first term on the right side of (4.10), it will be sufficient to show that the sum in (4.10) is also bounded.

By inspection of (4.6), it is clear that one maximum of $H$ will occur at $z=0$. In this case

$$
H(r, y, 0) \int_{0}^{y} e^{-z} z^{\alpha} d z=(1-r)^{-\alpha-1} \exp \left(\frac{-r y}{1-r}\right) \int_{0}^{y} e^{-z} z^{\alpha} d z .
$$

If $r \geqq \frac{1}{2}$, using the fact that the integral is less than $y^{\alpha+1} /(\alpha+1)$ shows that (4.11) is less than

$$
\frac{1}{\alpha+1}\left(\frac{y}{1-r}\right)^{\alpha+1} \exp \frac{-\frac{1}{2} y}{1-r}
$$

which is less than a constant depending only on $\alpha$. If $r<1 / 2$, using the fact that the integral is less than $\Gamma(\alpha+1)$ is sufficient.

No other maximum can occur for $0 \leqq z<(1-r)^{2} / 4 r y$ since $H$ is decreasing in this interval. It can be easily verified that $H$ is continuous at $z=(1-r)^{2} / 4 r y$; therefore, this can not be a maximum. If $z>(1-r)^{2} / 4 r y$, then

$$
\frac{\partial H}{\partial z}=\frac{-r z+(r y z)^{1 / 2}-(\alpha / 2+1 / 4)(1-r)}{e z(1-r)^{3 / 2}(4 r y z)^{\alpha / 2+1 / 4}} \exp \left(\frac{-r y+2(r y z)^{1 / 2}-r z}{1-r}\right) .
$$

This will equal 0 if

$$
\sqrt{ } z=\frac{\sqrt{ } y \pm(y-(1-r)(2 \alpha+1))^{1 / 2}}{2 \sqrt{ } r} .
$$

By observing the algebraic sign of $\partial H / \partial z$ it is clear that if there exists an $m \neq 0$ such that $H$ has a relative maximum at $z=m$, then

$$
\sqrt{ } m=\frac{\sqrt{ } y+(y-(1-r)(2 \alpha+1))^{1 / 2}}{2 \sqrt{ } r} .
$$


As mentioned at the beginning of this paragraph it is also true that

$$
m>(1-r)^{2} / 4 r y \text {. }
$$

If such a maximum occurred for $y<(1-r) / 3$, by (4.12) and the fact that $\alpha>-1$, $m$ would be less than $3(1-r) / 4 r$ and (4.13) would produce a contradiction. Therefore, if $m$ exists

$$
m \geqq(1-r) / 3 .
$$

Using (4.14) and the fact that $\alpha>-1$ on (4.12) then shows that

$$
y / 4 r \leqq m \leqq 9 y / 4 r .
$$

Now let $C$ denote a constant depending only on $\alpha$ but not necessarily the same at each occurrence. Using (4.15) in the nonexponential part of (4.6) shows that part is bounded by $C / y^{\alpha+1 / 2}(1-r)^{1 / 2}$ when $z=m$. The maximum value of the exponential part is $e^{y}$ and occurs at $z=y / r$. Combining these two bounds shows that

$$
L(r, y, m) \leqq C e^{y} / y^{\alpha+1 / 2}(1-r)^{1 / 2} .
$$

An estimate is also needed for $|m-y| r \mid$. This may be written as

$$
\left|\sqrt{ } m+(y / r)^{1 / 2}\right|\left|\sqrt{ } m-(y / r)^{1 / 2}\right|
$$

and by (4.15) the first part is bounded by $(5 / 2)(y / r)^{1 / 2}$. Using (4.12), the second part equals

$\left|\frac{\sqrt{ } y-(y-(1-r)(2 \alpha+1))^{1 / 2}}{2 \sqrt{ } r}\right|=\frac{(1-r)|2 \alpha+1|}{2 \sqrt{ } r\left[\sqrt{ } y+(y-(1-r)(2 \alpha+1))^{1 / 2}\right]} \leqq \frac{(1-r)|2 \alpha+1|}{2(r y)^{1 / 2}}$.

Combining these facts gives

$$
|m-y / r| \leqq C(1-r) / r .
$$

Now

$$
|y / r-y|=(1-r) y / r
$$

Combining (4.17) and (4.18) then gives

$$
|y-m| \leqq C(1-r)(y+1) / r .
$$

To complete the proof of Lemma 5 it is sufficient to show that

$$
H(r, y, m)\left|\int_{y}^{m} e^{-z} z^{\alpha} d z\right|
$$

is less than a constant depending only on $\alpha$ whenever $m$ exists. This will be done in three cases as indicated.

Case 1: $m<y$. Since the left side of (4.17) is greater than the left side of (4.18), $y \leqq C$. By (4.15) $m>y / 4$ and since $y / 4 r \leqq y, r \geqq 1 / 4$. Then using (4.16), (4.20) is less than $C y^{\alpha}|m-y| \mid y^{\alpha+1 / 2}(1-r)^{1 / 2}$. Using (4.14) and (4.19) gives the desired bound. 
Case 2: $m \geqq y$ and $y(1-r)<1 / 8$. In this case $r \leqq 1 / 4$ is impossible since this would imply $y \geqq 1 / 4$ by (4.14) and this would make $y(1-r) \geqq 3 / 16$. Therefore, $r>1 / 4$. Then using (4.15), $m<9 y$ and $\int_{y}^{m} e^{-z} z^{\alpha} d z \leqq C|m-y| e^{-y} y^{\alpha}$. Then using (4.16) and (4.19), (4.20) is less than

$$
\frac{C|m-y|}{(y(1-r))^{1 / 2}} \leqq C\left((y(1-r))^{1 / 2}+\left(\frac{1-r}{y}\right)^{1 / 2}\right) .
$$

The first term is bounded by the conditions of this case; the second is bounded by (4.14).

Case 3: $m \geqq y$ and $y(1-r) \geqq 1 / 8$. Then $y \geqq 1 / 8$ and $\int_{y}^{m} e^{-z} z^{\alpha} d z \leqq C e^{-y} y^{\alpha}$. This and (4.16) complete this case.

It is now simple to prove the main theorem concerning Poisson integrals for Laguerre expansions.

THEOREM 3. Let $f \in L^{p}\left(e^{-y} y^{\alpha}\right)$ on $[0, \infty)$ where $1 \leqq p \leqq \infty$ and $\alpha>-1$. Let $g(r, y)$ denote its Poisson integral (4.1), and $f^{*}(y)$ the function (2.1) with $d \mu(y)=e^{-y} y^{\alpha} d y$ and $I=[0, \infty)$. Then if $0 \leqq r<1$, there exists a constant $C$ depending only on $\alpha$ and $a$ constant $A_{p}$ depending only on $\alpha$ and $p$ such that:

(a) $|g(r, y)| \leqq C f^{*}(y)$,

(b) $\|g(r, y)\|_{p} \leqq\|f(y)\|_{p}, 1 \leqq p \leqq \infty$,

(c) $\|g(r, y)-f(y)\|_{p} \rightarrow 0$ as $r \rightarrow 1^{-}$for $1 \leqq p<\infty$,

(d) $\lim _{r \rightarrow 1^{-}} g(r, y)=f(y)$ almost everywhere, $1 \leqq p \leqq \infty$,

(e) $\left\|\sup _{r<1} g(r, y)\right\|_{p} \leqq A_{p}\|f\|_{p}, 1<p \leqq \infty$.

By (4.5), $K(r, y, z)$ is less than a constant times the function $L$ in Lemma 5. Part (a) then follows immediately from Corollary 2 . The rest of the proof is the same as that for Theorem 2 with (4.8) used instead of (3.7). $S$ in this case is the space of continuous functions with compact support on $(0, \infty)$. Again, given an $f \in S$ and an $\varepsilon>0$, $K(r, y, z)$ converges uniformly to 0 as $r \rightarrow 1^{-}$for $|y-z|>\varepsilon$ and $z$ in the support of $f$. This is proved by showing the fact for $H$; the exponential part of $H$ is the same as the exponential part of $P$ with all the variables replaced by their square roots.

To obtain the alternate Poisson integral define

$$
U(x, r)=T(x / \sqrt{ } 2, r)
$$

where $T$ is the function defined in (3.10). Then (3.9) becomes

$$
\int_{0}^{1} U(x, r) r^{n} d r=\exp (-\sqrt{ } n x)
$$

Since $f(x, y)$ is supposed to have the expansion $\sum a_{n} \exp (-\sqrt{ } n x) L_{n}^{\alpha}(x), f(x, y)$ will be defined by

(4.23) $f(x, y)=\int_{0}^{1} U(x, r) g(r, y) d r=\int_{0}^{\infty}\left(\int_{0}^{1} U(x, r) K(r, y, z) d r\right) f(z) e^{-z} z^{\alpha} d z$

for $x>0$. As in the Hermite case $f(x, y)$ is defined for almost every $y$ provided $f \in L^{1}\left(e^{-y} y^{\alpha}\right)$. 
COROLlaRY 4. If $f(y)$ has Laguerre expansion $\sum a_{n} L_{n}^{\alpha}(y)$, then for each $x>0$, $f(x, y)$ has the Laguerre expansion $\sum \exp (-\sqrt{ } n x) a_{n} L_{n}^{\alpha}(y)$. If $f(y)$ is in $L^{2}\left(e^{-y} y^{\alpha}\right)$, then $\sum \exp (-\sqrt{ } n x) a_{n} L_{n}^{\alpha}(y)$ converges absolutely to $f(x, y)$ for almost every $y$. The conclusions of Theorem 3 are valid if $g(r, y)$ is replaced by $f(x, y), r \rightarrow 1^{-}$by $x \rightarrow 0^{+}$ and $0 \leqq r<1$ by $x>0$. Furthermore, $f_{11}(x, y)+y f_{22}(x, y)+(\alpha+1-y) f_{2}(x, y)=0$.

The proof is the same as the proof of Corollary 3.

\section{REFERENCES}

1. S. Bochner and K. Chandrasekharan, Fourier transforms, Annals of Mathematics Studies No. 19, Princeton Univ. Press, Princeton, N. J., 1949.

2. G. H. Hardy, Summation of a series of polynomials of Laguerre, Addendum, J. London Math. Soc. 7 (1932), 192.

3. B. Muckenhoupt and E. M. Stein, Classical expansions and their relation to conjugate harmonic functions, Trans. Amer. Math. Soc. 118 (1965), 17-92.

4. I. P. Natanson, Theory of functions of a real variable, Vol. II, Ungar, New York, 1960.

5. H. Pollard, The mean convergence of orthogonal series. II, Trans. Amer. Math. Soc. 63 (1948), 355-67.

6. G. Szegö, Orthogonal polynomials, rev. ed., Amer. Math. Soc. Colloq. Publ., Vol. 23, Amer. Math. Soc., Providence, R. I., 1959.

7. G. N. Watson, Theory of Bessel functions, Cambridge Univ. Press, Cambridge, 1966.

8. A. Zygmund, Trigonometric series, 2nd ed., Vols. I, II, Cambridge Univ. Press, New York, 1959.

Rutgers, The STATE UNIVERSTT, New BRUnSWICK, NeW JeRSEY 